\section{Eyaculación precoz: revisión de la alteración y actualidades en el tratamiento}

Carrillo-Córdova LD, ${ }^{1}$ Rodríguez-Valle ED, ${ }^{1}$ Rodríguez-Robles JA, ${ }^{1}$ VitarSandoval J, ${ }^{1}$ Carrillo-Córdova JR, ${ }^{2}$ Obregón-Aguilar $A,{ }^{1,3}$ y colaboradores.

\begin{abstract}
Resumen
La eyaculación precoz es el trastorno sexual masculino más común, puede clasificarse en eyaculación precoz permanente o adquirida, cuyo mecanismo de origen aún se desconoce. Durante la actividad sexual pueden identificarse cuatro eventos en la respuesta normal: 1) erección, 2) emisión, 3) eyaculación y 4) orgasmo. Cuando la eyaculación precoz genera pocos síntomas, el tratamiento debe limitarse a la orientación psicosexual. Varias técnicas de comportamiento han demostrado beneficio en el tratamiento de la disfunción eréctil; sin embargo, no deben considerarse como protocolos de primera línea o tratamiento único en pacientes con eyaculación precoz permanente, en quienes la farmacoterapia es la base del mismo. La eyaculación precoz es una alteración frecuente, con consecuencias psicosociales considerables. El tratamiento se enfoca en aumentar el tiempo del acto sexual y debe individualizarse en cada paciente.
\end{abstract}

PALABRAS CLAVE: eyaculación precoz, tratamiento médico, tratamiento quirúrgico.

Rev Mex Urol. 2017 Jul-Aug;77(4):328-335.

\section{Premature ejaculation: A review of the pathology and treatment update}

Carrillo-Córdova LD, ${ }^{1}$ Rodríguez-Valle ED, ${ }^{1}$ Rodríguez-Robles JA, ${ }^{1}$ VitarSandoval J, ${ }^{1}$ Carrillo-Córdova JR, ${ }^{2}$ Obregón-Aguilar A, ${ }^{1,3}$ et al.

\footnotetext{
Abstract

It is the most common male sexual disorder and can be classified as lifelong or acquired premature ejaculation, but the mechanism causing it is still unknown. Four events are identified in as normal response during sexual activity: 1) erection, 2) emission, 3) ejaculation, and 4) orgasm. When premature ejaculation produces few symptoms,
}

\begin{abstract}
${ }^{1}$ Departamento de Urología, Hospital General de México Dr. Eduardo Liceaga, Ciudad de México, México.

${ }^{2}$ Departamento de Cirugía Plástica y Reconstructiva, Hospital General Dr. Manuel Gea González, Ciudad de México, México.

${ }^{3}$ Estudiante de pregrado, Universidad Nacional Autónoma de México.
\end{abstract}

Recibido: diciembre 2016

Aceptado: junio 2017

Correspondencia

Dr. Luis Daniel Carrillo Córdova

carrillocor@gmail.com

Este artículo debe citarse como

Carrillo-Córdova LD, Rodríguez-Valle ED, RodríguezRobles JA, y col. Eyaculación precoz. Revisión de la alteración y actualidades en el tratamiento. Rev Mex Urol. 2017 jul-agos;77(4):328-335.

DOI: https://doi.org/10.24245/revmexurol.v77i4.1195 
treatment should be limited to psychosexual counseling. Several behavioral techniques have been shown to be beneficial in erectile dysfunction treatment, but they should not be considered first-line or sole treatment in patients with lifelong premature ejaculation. Those patients require drug-based management. Premature ejaculation is a frequent pathology with considerable psychosocial consequences. Treatment is directed at improving the duration of the sexual act and should be individualized for each patient.

KEYWORDS: Premature ejaculation; Medical treatment; Surgical treatment

\author{
${ }^{1}$ Urology Department, Hospital General de \\ Mexico Dr. Eduardo Liceaga, Mexico City. \\ ${ }^{2}$ Reconstructive and Plastic Surgery De- \\ partment, Hospital General de Mexico Dr. \\ Eduardo Liceaga, Mexico City. \\ ${ }^{3}$ Estudiante de pregrado, Universidad \\ Nacional Autónoma de México. \\ Correspondence \\ Dr. Luis Daniel Carrillo Córdova \\ carrillocor@gmail.com
}

\section{ANTECEDENTES}

La eyaculación precoz es una disfunción sexual que genera angustia considerable para el paciente y su pareja. ${ }^{1}$ Hasta el momento no existe una definición aceptada de manera universal. La disfunción sexual, la infertilidad y los síntomas de la vía urinaria inferior representan factores de riesgo reconocidos para padecer eyaculación precoz. ${ }^{2}$ El tratamiento de la eyaculación precoz ha evolucionado con el paso de los años. La farmacoterapia es el tratamiento de primera línea; sin embargo, existen opciones adicionales como: procedimientos quirúrgicos, terapias conductuales y psicológicas.

El objetivo de este estudio es describir las opciones de tratamiento actuales de la eyaculación precoz.

\section{EPIDEMIOLOGÍA}

La eyaculación precoz es el trastorno sexual masculino mas común, cuya prevalencia varía de $20-30 \%$ en los hombres que experimentan disfunción eréctil; sin embargo, esta alteración suele subdiagnosticarse debido a la búsqueda limitada de asesoría médica por cuestiones sociales. ${ }^{3,4}$

\section{CLASIFICACIÓN}

La eyaculación precoz se clasifica en dos grandes grupos:

1. Eyaculación precoz permanente. Se inicia desde la primera experiencia sexual y persiste a lo largo de la vida. La eyaculación ocurre con demasiada rapidez, incluso antes de la penetración vaginal o como un tiempo de latencia de la eyaculación intravaginal (IELT) menor a 1-2 minutos. El tiempo de latencia de la eyaculación intravaginal se define como el tiempo entre la penetración vaginal y la eyaculación intravaginal.

2. Eyaculación precoz adquirida. Se distingue por evolución gradual en hombres que previamente experimentaron una eyaculación normal. El tiempo para la eyaculación es corto, pero no suele ser tan rápido como en la eyaculación permanente. ${ }^{5,6}$ 


\section{FISIOPATOLOGÍA}

El mecanismo por el que se origina la eyaculación precoz sigue sin conocerse; sin embargo, existen teorías que pueden explicar la alteración; por ejemplo, durante la actividad sexual pueden identificarse cuatro eventos en la respuesta normal: 1) erección, 2) emisión, 3) eyaculación y 4) orgasmo. La eyaculación es regulada por un reflejo espinal que, a su vez, es controlado centralmente por receptores sensoriales y vías nerviosas aferentes, áreas motoras y sensoriales, y el generador espinal de la eyaculación, ubicado entre las vértebras T12-L2 de la médula espinal. Este complejo sistema de señalización incluye neurotransmisores serotoninérgicos, dopaminérgicos, oxitocinérgicos y receptores opioides. ${ }^{7,8}$ El sistema serotoninérgico inhibe el reflejo eyaculatorio en el hipotálamo. ${ }^{9}$ La vía dopaminérgica estimula la eyaculación. ${ }^{10}$ En pacientes con eyaculación precoz se han determinado bajas concentraciones de prolactina, ${ }^{11}$ además de alta prevalencia en sujetos con hipertiroidismo; de manera opuesta, se ha observado eyaculación retrasada en pacientes con hipotiroidismo. ${ }^{12}$

\section{TRATAMIENTO}

Cuando la eyaculación precoz produce pocos síntomas, el tratamiento debe limitarse a orientación psicosexual. ${ }^{6}$ Varias técnicas han demostrado beneficio en el tratamiento de la disfunción eréctil; sin embargo, no deben considerarse como primera línea o tratamiento único en pacientes con eyaculación precoz permanente, ${ }^{5}$ en quienes la farmacoterapia es la base del mismo.

\section{Fármacos prescritos por vía oral}

a. Inhibidores selectivos de la recaptación de serotonina (ISRS)

Una hipótesis del origen de la eyaculación precoz es la disfunción de los receptores $5-\mathrm{HT} .{ }^{13}$ Los
ISRS inhiben la función de los trasportadores de serotonina presinápticos y somatodendríticos, con lo que aumenta la neurotransmisión total de 5-HT central y la activación de los receptores postsinápticos $5-\mathrm{HT},{ }^{14}$ específicamente 5 -HT1B y 5 -HT2C para generar un efecto inhibitorio global en la eyaculación. ${ }^{15}$ Los ISRS de consumo diario son la primera opción de tratamiento en pacientes con eyaculación precoz; estos incluyen: paroxetina (20-40 mg/d), sertralina (25-200 $\mathrm{mg} / \mathrm{d})$ y fluoxetina $(10-60 \mathrm{mg} / \mathrm{d}) .^{13}$

El efecto de los ISRS en la eyaculación precoz se ha observado pocos días después de iniciar la medicación; sin embargo, la mayoría de los pacientes percibe un aumento significativo en el tiempo de eyaculación, entre 1 y 2 semanas después. ${ }^{16}$ El tratamiento a demanda es menos efectivo que el indicado con dosis diarias, pero puede combinarse con dosis bajas para disminuir los efectos adversos..$^{17}$ Un estudio reportó que la paroxetina es superior a la fluoxetina, clomipramina y sertralina. Los efectos secundarios más comunes incluyen: fatiga, somnolencia, bostezos, náuseas, vómito, sequedad de boca, diarrea, traspiración, disminución de la libido, anorgasmia, aneyaculación y disfunción eréctil. ${ }^{13}$

\section{Dapoxetina}

Es un ISRS potente, diseñado específicamente para el tratamiento de la eyaculación precoz. Su perfil farmacológico apoya el papel del tratamiento bajo demanda. ${ }^{18-20}$ La dapoxetina impide el trasporte, en lugar de la recaptación de serotonina. Comparada con los ISRS clásicos, alcanza su concentración máxima en una hora y después se elimina rápidamente del organismo. ${ }^{21}$ Un estudio demostró que la dapoxetina, en dosis de 30 y $60 \mathrm{mg}$, aumentó significativamente el tiempo de latencia de eyaculación intravaginal versus placebo. ${ }^{18}$ Otro estudio evidenció que el control de la eyaculación mejoró en 51 y 58\% de los pacientes a quienes se administraron 30 y $60 \mathrm{mg}$ de dapoxentina, respectivamente. Ambos 
protocolos fueron efectivos en la primera dosis. ${ }^{20}$ Los efectos adversos más frecuentes fueron náuseas, mareos, diarrea y cefalea.

\section{Tramadol}

El tramadol es un potente analgésico opioide de acción central que se prescribe en el tratamiento del dolor. Ejerce sus efectos analgésicos al unirse con receptores centrales opioides del dolor $\mathrm{MU}$ (m) e inhiben la recaptación de serotonina y norepinefrina. ${ }^{22}$ Se ha demostrado que el tramadol funciona como antagonista del receptor 5-HT2C y su efectividad en el tratamiento de la eyaculación precoz se debe a la neuromodulación de los receptores de serotonina y norepinefrina. ${ }^{23,24}$

El tramadol aumenta significativamente el tiempo de latencia de eyaculación intravaginal comparado con el placebo, por ejemplo: 1.6 veces para placebo, 2.4 veces con dosis de $62 \mathrm{mg} \mathrm{y}$ 2.5 veces con protocolo de $89 \mathrm{mg}$ de tramadol. ${ }^{24}$ A diferencia de otros tratamientos prescritos en pacientes con eyaculación precoz, el tramadol es capaz de generar abuso y dependencia; sin embargo, esos episodios son raros. ${ }^{25}$ El tramadol no debe combinarse con un ISRS, debido al riesgo de síndrome de serotonina. ${ }^{26}$

\section{b. Inhibidores de la fosfodiesterasa tipo 5 (PDE5i)}

Los PDE5i son fármacos indicados para el tratamiento de la disfunción eréctil. Su prescripción en pacientes con eyaculación precoz aún se discute, debido a la falta de evidencia del retraso de la eyaculación. ${ }^{27}$ La hipótesis del mecanismo de los PDE5i en la eyaculación precoz incluye el aumento de la liberación de óxido nítrico, reducción del tono simpático y dilatación del músculo liso del conducto deferente y las vesículas seminales. ${ }^{28}$ Sin embargo, el tiempo de latencia de eyaculación intravaginal no se incrementa significativamente, pero aumenta la confianza, percepción del control eyaculatorio y satisfacción sexual, además de disminuir la ansiedad y el tiempo para lograr una segunda erección. ${ }^{13}$ Por lo tanto, los PED5i no deben indicarse en pacientes con eyaculación precoz y función eréctil normal.

\section{c. Antagonistas del adrenorreceptor alfa-1}

Estos medicamentos son el patrón de referencia para disminuir los síntomas de la vía urinaria inferior de pacientes con enfermedad prostática. Diversos estudios informan que uno de los principales efectos adversos es la disfunción eyaculatoria, ${ }^{29}$ que inicialmente se pensaba su origen se relacionaba con eyaculación retrógrada; sin embargo, estudios recientes demuestran que se debe a la deficiencia de contracción de las vesículas seminales. ${ }^{29,30}$ Los bloqueadores alfa-1 más prescritos en el tratamiento de la eyaculación precoz incluyen: tamsulosina, silodosina, terazosina y alfuzosina, que han demostrando un aumento estadísticamente significativo del tiempo de latencia de la eyaculación intravaginal. ${ }^{31}$ Con dosis terapéuticas de referencia son raros los efectos secundarios, pero puede haber disminución del volumen eyaculatorio, eyaculación seca, anorgasmia, sequedad de boca, congestión nasal, somnolencia e hipotensión ortostática. ${ }^{32}$

\section{d. Antidepresivos tricíclicos}

Al igual que los ISRS, los antidepresivos tricíclicos ejercen su efecto al inhibir el trasporte de serotonina y norepinefrina, aumentar la concentración de serotonina y norepinefrina en la hendidura sináptica y retrasar la eyaculación a través de la actividad en los receptores $5-\mathrm{HT}^{33}$ Un estudio demostró que la clomipramina administrada diariamente tuvo mayor efecto en el tiempo de latencia de la eyaculación intravaginal que la fluoxetina o la sertralina. ${ }^{34}$ La principal desventaja de los antidepresivos tricíclicos son 
sus efectos secundarios: fatiga, náuseas, sequedad de boca, hipotensión, enrojecimiento y mareo.

\section{Tratamiento tópico}

Anestésicos locales

La respuesta eyaculatoria consiste en una entrada neuronal aferente, Ilevada por el nervio pudendo y activada por la estimulación del pene. Posteriormente existe una salida neuronal eferente, estimulada por los nervios simpáticos de los ganglios simpáticos paraespinosos entre las vértebras T10-T12 a los órganos reproductores masculinos para activar la eyaculación. La eyaculación precoz aparece como respuesta hipersensible a la estimulación del pene. ${ }^{35,36}$

Los anestésicos locales estabilizan la membrana, inhiben los canales de sodio controlados por voltaje, con lo que disminuye la trasmisión sináptica e hipersensibilidad neuronal, y aumenta el umbral eyaculatorio. ${ }^{37}$ La crema con lidocaína-prilocaína (EMLA) se aplica 2030 minutos antes del coito; algunos estudios señalan que aumenta significativamente el tiempo de latencia de eyaculación intravaginal. Uno de los principales efectos secundarios incluye entumecimiento del pene (debe usarse con condón para evitar su difusión a la pared vaginal). ${ }^{38,39}$

La formulación en aerosol de lidocaína y prilocaína (TEMPE) no penetra la piel queratinizada y diminuye la mayor parte de los efectos secundarios. ${ }^{40}$ Existe otra presentación: lidocaína en aerosol (PROMESCENT) sin prilocaína, que se aplica antes del coito. Este producto desensibiliza el glande y aumenta el tiempo de latencia de la eyaculación intravaginal; sin embargo, se requieren estudios adicionales que confirmen este resultado. ${ }^{41}$ Existe un agente anestésico tópico elaborado con extractos de nueve hierbas; se aplica en el glande 1 hora antes del coito y se lava inmediatamente antes de iniciar el acto sexual. Un ensayo demostró que la aplicación de 0.2 g aumenta significativamente el tiempo de latencia de la eyaculación intravaginal versus placebo. ${ }^{42}$

\section{Tratamiento quirúrgico}

El tratamiento quirúrgico ha adquirido popularidad en países asiáticos; sin embargo, aún no ha logrado su aceptación en el resto del mundo. Actualmente se han descrito tres abordajes quirúrgicos: neurectomía del nervio dorsal, aumento del glande con ácido hialurónico y circuncisión. ${ }^{43}$

\section{Neurectomía del nervio dorsal}

Un estudio realizado en Corea del Sur comparó la neurectomía del nervio dorsal versus circuncisión, con seguimiento a 6 meses, y mostró que en el grupo intervenido de neurectomía aumentó el tiempo de latencia de eyaculación intravaginal 2.7 veces y la percepción en el control eyaculatorio. Los pacientes circuncidados no mostraron cambios significativos. ${ }^{44}$ Resultados similares se observaron en estudios que analizaron la radiofrecuencia pulsada en el nervio dorsal, así como la crioablación guiada por tomografía. ${ }^{45,46}$ Sin embargo, no existen datos a largo plazo de la seguridad y eficacia de la neurectomía del nervio dorsal en el tratamiento de la eyaculación precoz, además de que los estudios disponibles han evaluado un tamaño limitado de pacientes. Debe tenerse cautela con esta técnica, debido a que es un procedimiento invasivo e irreversible. No existen estudios de seguimiento a largo plazo que describan las complicaciones de la neurectomía del nervio dorsal; sin embargo, un estudio esclarece que la complicación más común es la recurrencia de eyaculación precoz en aproximadamente $10 \%$ de los casos. ${ }^{43}$ 


\section{Aumento del glande con ácido hialurónico}

El principio de este tratamiento es la inyección de gel de ácido hialurónico en el glande, con la finalidad de crear una barrera física permanente entre los receptores del nervio dorsal hipersensible. Algunos estudios reportan 2.9 veces más de aumento en el tiempo de latencia de la eyaculación intravaginal 6 meses después del procedimiento. ${ }^{47,48}$ Informes recientes indican posibles complicaciones como: reacciones granulomatosas por cuerpo extraño y necrosis isquémica. ${ }^{49}$ Sin embargo, no existen suficientes estudios para garantizar su seguridad y eficacia.

\section{Circuncisión}

El prepucio se encuentra inervado con neuronas sensoriales, lo que resulta en alta sensibilidad..$^{50}$ La explicación más acertada de la circuncisión es que durante el acto quirúrgico también se extirpan muchos de estos receptores sensoriales, lo que disminuye la sensibilidad del glande. Sin embargo, la mayor parte de los estudios no muestran aumento de la función sexual después del procedimiento. ${ }^{51,52}$

\section{Terapia psicológica}

Existen dos clases principales de terapia: la conductual y la psicológica. Esta última consiste en asesoramiento psicosexual para hombres y sus parejas, donde se tratan problemas psicológicos e interpersonales. La terapia conductual incluye técnicas físicas para ayudar a los pacientes a desarrollar habilidades para retrasar la eyaculación y mejorar la autoconfianza.

La psicoterapia actual para el tratamiento de la eyaculación precoz debe implementarse como coadyuvante y no como protocolo de primera línea. Algunos estudios sugieren tasas de éxito de $50-60 \%$ a corto plazo, pero no se mantienen a largo plazo. ${ }^{53,54} \mathrm{Sin}$ embargo, existe evidencia que en combinación con tratamientos farmacológicos se amplían y optimizan sus efectos. ${ }^{55}$

Los objetivos del tratamiento psicológico incluyen: aprendizaje de técnicas para controlar, retrasar, o ambas, la eyaculación, ganar confianza en el desempeño sexual, disminución de la ansiedad de rendimiento, superar las barreras de intimidad, resolver problemas interpersonales que precipiten y mantengan la disfunción, identificar sentimientos-pensamientos que interfieren con la función sexual y aumentar la comunicación. ${ }^{56}$

Como parte de la terapia conductual se encuentran las siguientes técnicas:

La técnica de "detener-empezar" (stop-start) consiste en que el hombre o su pareja estimulan el pene hasta que sienta el impulso de eyacular y luego se detiene hasta que pase la sensación. Esto se repite en múltiples ocasiones antes de permitir que se produzca la eyaculación. ${ }^{57} \mathrm{El}$ objetivo es conocer la sensación de excitación, con la finalidad de mejorar el control de ésta.

En la técnica de "exprimir" (squeeze) la pareja del paciente estimula el pene hasta que sobreviene el impulso de eyacular, posteriormente presiona el glande hasta que pase la sensación, de igual manera, esto se repite varias veces antes de permitir que se produzca la eyaculación. ${ }^{57}$ Por último, los ejercicios para rehabilitación muscular del piso pélvico también pueden ayudar al control eyaculatorio. ${ }^{58}$

\section{CONCLUSIÓN}

La eyaculación precoz es una alteración frecuente que genera consecuencias psicosociales considerables. El tratamiento se enfoca en incrementar el tiempo del acto sexual y debe individualizarse en cada paciente. En 
los casos leves puede indicarse terapia conductual, con la que se han obtenido resultados favorables; los casos más graves requieren la combinación de tratamiento farmacológico oral y tópico asociado con terapia psicológica. El tratamiento quirúrgico se implementa en casos seleccionados; sin embargo, sigue siendo motivo de discusión en todo el mundo.

\section{REFERENCIAS}

1. Rowland DL, Patrick DL, Rothman M, et al. The psychological burden of premature ejaculation. J Urol 2007;177:106570.

2. Jannini EA, Ciocca G, Limocin E, Mollaioli D, et al. Premature ejaculation: old story, new insights. Fertil. Steril. 2015;104:1061-1073.

3. Patrick DL, Althof SE, Pryor JL, et al. Premature ejaculation: an observational study of men and their partners. J Sex Med 2005;2:358-67.

4. Waldinger MD. Relevance of an evidence-based ejaculation time cutoff point for neurobiological research of premature ejaculation. J. Comp. Neurol. 2005;493:46-50.

5. McMahon CG, Althof SE, Waldinger MD, et al. An evidencebased definition of lifelong premature ejaculation: report of the International Society for Sexual Medicine (ISSM) ad hoc committee for the definition of premature ejaculation. J Sex Med 2008;5:1590-606.

6. Waldinger MD. Premature ejaculation: state of the art. Urol Clin North Am 2007;34:591-9, vii-viii.

7. Giuliano F. Neurophysiology of erection and ejaculation. J Sex Med 2011;8(Suppl. 4):310-315.

8. Simoes Paco J, Jorge Pereira B. New therapeutic perspectives in premature ejaculation. Urology 2016;88:87-92.

9. Porst H. An overview of pharmacotherapy in premature ejaculation. J Sex Med 2011;8(Suppl. 4):335-341.

10. Giuliano F, Clement P. Pharmacology for the treatment of premature ejaculation. Pharmacol Rev 2012;64:621-644.

11. Corona G, et al. Hypoprolactinemia: a new clinical syndrome in patients with sexual dysfunction. J Sex Med 2009;6:1457-1466

12. Sansone A, et al. Hormonal correlations of premature ejaculation. Endocrine 2015;49:333-338.

13. Wespes EC, Eardley I, Giuliano F, et al. Guidelines on Male Sexual Dysfunction: erectile dysfunction and premature ejaculation. European Association of Urology, Arnhem, 2013.

14. Olivier B, van Oorschot R, Waldinger MD. Serotonin, serotonergic receptors, selective serotonin reuptake inhibitors and sexual behaviour. Int. Clin. Psychopharmacol. 1998; 13(Suppl 6): S9-14.
15. Giuliano F. 5-Hydroxytryptamine in premature ejaculation: opportunities for therapeutic intervention. Trends Neurosci. 2007;30:79-84.

16. Waldinger MD. Premature ejaculation: definition and drug treatment. Drugs 2007;67:547-68.

17. Waldinger MD, Zwinderman AH, Olivier B. On-demand treatment of premature ejaculation with clomipramine and paroxetine: a randomized, double-blind fixed-dose study with stopwatch assessment. Eur Urol 2004;46:510-6.

18. Pryor JL, Althof SE, Steidle C, et al. Efficacy and tolerability of dapoxetine in treatment of premature ejaculation: an integrated analysis of two double-blind, randomised controlled trials. Lancet 2006;368:929-37.

19. McMahon C, Kim SW, Park NC, et al. Treatment of premature ejaculation in the Asia-Pacific region: results from a phase III double-blind, parallel-group study of dapoxetine. J Sex Med 2010;7:256-68.

20. Buvat J, Tesfaye F, Rothman M, et al. Dapoxetine for the treatment of premature ejaculation: results from a randomized, double-blind, placebo-controlled phase 3 trial in 22 countries. Eur Urol 2009;55:957-67.

21. Gur S, Kadowitz PJ, Sikka SC, Current therapies for premature ejaculation. Drug Discov Today 2016;21:1147-1154.

22. Leppert $\mathbf{W}$. Tramadol as an analgesic for mild to moderate cancer pain. Pharmacol Rep 2009;61:978-92.

23. Ogata J, Minami K, Uezono $Y$, et al. The inhibitory effects of tramadol on 5-hydroxytryptamine type $2 \mathrm{C}$ receptors expressed in Xenopus oocytes. Anesth. Analg. 2004; 98:1401-6.

24. Bar-Or D, Salottolo KM, Orlando A, Winkler JV; Tramadol ODT Study Group. A randomized double-blind, placebocontrolled multicenter study to evaluate the efficacy and safety of two doses of the tramadol orally disintegrating tablet for the treatment of premature ejaculation within less than 2 minutes. Eur Urol 2012;61:736-43.

25. Kirby EW, Carson CC, Coward RM. Tramadol for the management of premature ejaculation: a timely systematic review. Int J Impot Res 2015;27:121-7.

26. Takeshita J, Litzinger MH. Serotonin syndrome associated with tramadol. Prim Care Companion J Clin Psychiatry 2009;11:273.

27. Jannini EA, McMahon C, Chen J, Aversa A, Perelman M. The controversial role of phosphodiesterase type 5 inhibitors in the treatment of premature ejaculation. J Sex Med 2011;8:2135-2143.

28. McMahon CG, McMahon CN, Leow LJ, Winestock CG. Efficacy of type- 5 phosphodiesterase inhibitors in the drug treatment of premature ejaculation: a systematic review. Br J Urol 2006;98:259-72.

29. Debruyne FM. Alpha blockers: are all created equal? Urology 2000;56(5 Suppl 1):20-2.

30. Hisasue SI, Furuya R, Itoh N, Kobayashi K, Furuya S, Tsukamoto T. Ejaculatory disorder caused by alpha-1 adrenoceptor antagonists is not retrograde ejaculation but a loss of seminal emission. Int. J Urol 2006;13:1311-6. 
31. Akin Y, Gulmez H, Ates M, Bozkurt A, Nuhoglu B. Comparison of alpha blockers in treatment of premature ejaculation: a pilot clinical trial. Iran. Red Crescent Med J 2013;15:e13805.

32. Debruyne FM, Van der Poel HG. Clinical experience in Europe with uroselective alpha 1-antagonists. Eur Urol 1999;36(Suppl 1):54-58.

33. Gillman PK. Tricyclic antidepressant pharmacology and therapeutic drug interactions updated. $\mathrm{Br} J$ Pharmacol 2007;151:737-48.

34. Kim SC, Seo KK. Efficacy and safety of fluoxetine, sertraline and clomipramine in patients with premature ejaculation: a double-blind, placebo controlled study. J Urol 1998;159:425-7.

35. Xin ZC, Chung WS, Choi YD, Seong DH, Choi YJ, Choi HK. Penile sensitivity in patients with primary premature ejaculation. J Urol 1996;156:979-81.

36. Guyton and Hall Textbook of Medical Physiology. Philadelphia: Elsevier, 2016.

37. Yagiela JA. Vasoconstrictor agents for local anesthesia. Anesth Prog 1995;42:116-20.

38. Atikeler MK, Gecit I, Senol FA. Optimumusage of prilocainelidocaine cream in premature ejaculation. Andrologia 2002;34:356-9.

39. Busato W, Galindo CC. Topical anaesthetic use for treating premature ejaculation: a double-blind, randomized, placebo-controlled study. BJU Int 2004;93:1018-21.

40. Henry R, Morales A, Wyllie MG. TEMPE: topical eutecticlike mixture for premature ejaculation. Expert Opin. Drug Deliv. 2008;5:251-61.

41. Gilbert R. Promescent. Absorption Pharmaceuticals, LLC (Online), 2015. (Cited 29 Dic 2016.) Available from URL: https://www.promescent.com/about-promescent/ promescent-and-psd-502.

42. Choi HK, Jung GW, Moon KH, et al. Clinical study of SScream in patients with lifelong premature ejaculation. Urology 2000;55:257-61.

43. Yang DY, Ko K, Lee WK, et al. Urologist's practice patterns including surgical treatment in the management of premature ejaculation: A Korean nationwide survey. World J Mens Health 2013;31:226-31.

44. Zhang GX, Yu LP, Bai WJ, et al. Selective resection of dorsal nerves of penis for premature ejaculation. Int J Androl 2012;35:873-9.
45. Basal S, Goktas S, Ergin A, et al. A novel treatment modality in patients with premature ejaculation resistant to conventional methods: the neuromodulation of dorsal penile nerves by pulsed radiofrequency. J Androl 2010;31:126-30.

46. David-Prologo J, Snyder LL, Cherullo E, et al. Percutaneous CT-guided cryoablation of the dorsal penile nerve for treatment of symptomatic premature ejaculation. J Vasc Interv Radiol 2013;24:214-9.

47. Kim JJ, Kwak TI, Jeon BG, et al. Effects of glans penis augmentation using hyaluronic acid gel for premature ejaculation. Int J Impot Res 2004;16:547-51.

48. Kwak $\mathrm{TI}$, Jin $\mathrm{MH}, \mathrm{Kim} \mathrm{JJ}$, et al. Long-term effects of glans penis augmentation using injectable hyaluronic acid gel for premature ejaculation. Int J Impot Res 2008;20:425-8.

49. Moon du G, Kwak TI, Kim JJ. Glans penis augmentation using hyaluronic acid gel as an injectable filler. World J Mens Health 2015;33:50-61.

50. Tuncali D, Bingul F, Talim B, et al. Histologic characteristics of the human prepuce pertaining to its clinical behavior as a dual graft. Ann Plast Surg 2005;54:191-5.

51. Tian Y, Liu W, Wang JZ, et al. Effects of circumcision on male sexual functions: a systematic review and metaanalysis. Asian J Androl 2013;15:662-6.

52. Cortes-Gonzalez JR, Arratia-Maqueo JA, Martinez-Montelongo $\mathrm{R}$, et al. Does circumcision affect male's perception of sexual satisfaction? Arch Esp Urol 2009;62:733-6.

53. Metz ME, Pryor JL, Nesvacil LJ, Abuzzahab F Sr, Koznar J. Premature ejaculation: a psychophysiological review. J Sex Marital Ther 1997;23:3-23.

54. De Amicis LA, Goldberg DC, LoPiccolo J, Friedman J, Davies L. Clinical follow-up of couples treated for sexual dysfunction. Arch Sex Behav 1985;14:467-89.

55. Perelman MA. A new combination treatment for premature ejaculation: a sex therapist's perspective. J Sex Med 2006;3:1004-12.

56. Althof SE. Psychological approaches to the treatment of rapid ejaculation. The JMHG 2006;3:180-6.

57. Melnik T, Althof S, Atallah AN, Puga MEDS, Glina S, Riera R. Psychosocial interventions for premature ejaculation. Cochrane Database Syst Rev 2011;(8):CD008195.

58. Pastore AL, Palleschi G, Leto A, Pacini L, lori F, Leonardo C, et al. A prospective randomized study to compare pelvic floor rehabilitation and dapoxetine for treatment of lifelong premature ejaculation. Int J Androl 2012;35:528-33. 